\title{
Long-term remission of disseminated parathyroid cancer following immunotherapy
}

\author{
Marta Sarquis ${ }^{1}$ - Stephen J. Marx ${ }^{2}$ - Albert Beckers ${ }^{3}$ - Arthur R. Bradwell ${ }^{4}$ William F. Simonds ${ }^{5}$.

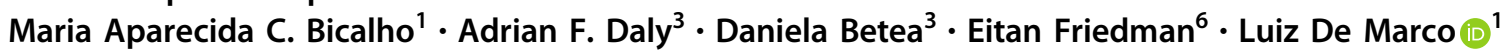

Received: 6 August 2019 / Accepted: 9 November 2019

(c) Springer Science+Business Media, LLC, part of Springer Nature 2019

\begin{abstract}
Purpose Parathyroid cancer is a rare tumor associated with poor prognosis particularly when disseminated. While chemotherapy and/or radiotherapy are of no clinical value in disseminated disease, immunotherapy should be considered.

Subject and results A patient with $C D C 73$-associated metastatic parathyroid carcinoma was treated with combined antihPTH immunotherapy and surgery.

Conclusions Following five courses of anti-hPTH immunotherapy and subsequent surgery, a 12-year long remission of disseminated parathyroid cancer is reported. This case further supports the ever-expanding spectrum of cancers that may benefit from immunotherapy.
\end{abstract}

Keywords Parathyroid $\cdot$ Cancer Immunotherapy

\section{Introduction}

Parathyroid carcinoma is a rare malignancy encountered in less than $1 \%$ of patients with primary hyperparathyroidism $\left(1^{\circ} \mathrm{HPT}\right)[1-4]$. Patients with parathyroid carcinoma typically present with larger tumors and with higher PTH and calcium levels than other forms of $1^{\circ} \mathrm{HPT}$. Differentiating histologically between parathyroid carcinoma and atypical

Luiz De Marco

Ldemarco@ufmg.br

1 Department of Surgery, Faculdade de Medicina, Universidade Federal de Minas Gerais, Av Alfredo Balena 190, Belo Horizonte 30130-100, Brazil

2 Section on Genetics and Endocrinology (SEGEN), Eunice Kennedy Shriver National Institute of Child Health and Human Development (NICHD), Bethesda, MD, USA

3 Department of Endocrinology, Centre Hospitalier Universitaire de Liège, Liège Université, Liège, Belgium

4 College of Medical and Dental Sciences, University of Birmingham, Birmingham, UK

5 Metabolic Diseases Branch, National Institute of Diabetes and Digestive and Kidney Diseases, National Institutes of Health, Bethesda, MD, USA

6 The Suzanne Levy Gertner Oncogenetics Unit, Chaim Sheba Medical Center, Tel-Hashomer, Israel parathyroid adenoma is challenging, as the histopathology of these tumors can be equivocal. A nontruncated aminoterminal variant of PTH is massively overproduced in parathyroid carcinoma, but only rarely in severe $1^{\circ} \mathrm{HPT}$ and helps in distinguishing between these tumor types [5].

The overall 5- and 10-year survival rates in parathyroid carcinoma range from 50-90\% [3]. Distant metastases substantially worsen survival rates compared with locally invasive parathyroid carcinoma (hazards ratio $3.5 ; 95 \%$ CI 1.6-7.64 years) [6, 7]. En bloc resection is still the only option for achieving cure but treatment of disseminated disease is challenging [2, 3, 6, 7], even when aimed only to control hypercalcemia. Radiotherapy and chemotherapy for disseminated parathyroid carcinoma confer minimal survival improvement or hypercalcemia control [2, 3, 6, 7]. In addition, while the calcimimetic agent cinacalcet has been approved for use in parathyroid cancer to lower PTH and calcium levels $[3,8]$ it has no proven antitumoral effect. Thus, there is a need for novel treatment options for metastatic parathyroid carcinoma.

Three cases of disseminated parathyroid carcinoma were previously reported to have been treated with antiPTH immunotherapy [9-11]. Here we report disseminated parathyroid carcinoma treated with anti-hPTH immunotherapy and surgery that led to a 12-year disease-free remission. 


\section{Subject and results}

The Brazilian proband, born in 1982, was diagnosed with $1^{\circ}$ HPT at age 18 and underwent a left inferior parathyroidectomy, with no evidence of parathyroid carcinoma at that time. She was lost to postsurgical follow up. Six years later, she was diagnosed with hypercalcemia, nephrolithiasis, and osteopenia. After two additional cervical explorations, her calcium and PTH levels remained elevated (Fig. 1). In August 2006 the patient underwent exploration of the retropharyngeal, retroesophageal space, right atria of the brachiocephalic trunk, left subclavian and left jugular vein and mediastinum, and removal of the left thyroid lobe. A small left lateral pleural nodule was palpable but was not biopsied for technical reasons. No parathyroid tissue was found in surgically resected samples, and her calcium and PTH remained elevated.

Genotyping showed a CDC73 germline mutation (c.686_688delAGAG) [12, 13]. In April 2007, she was referred to the National Institutes of Health (NIH) for management of persistent hyperparathyroidism. On admission, intact PTH was $582 \mathrm{pg} / \mathrm{mL}$ (normal range 16-87), ionized calcium $1.93 \mathrm{mmol} / \mathrm{L}$ (1.22-1.38), and total calcium $12.6 \mathrm{mg} / \mathrm{dL}$ (8.6-10.5). Presurgical chest CT showed a $2.5 \mathrm{~cm}$ left apical lung mass positive on $99 \mathrm{mTc}-$ SESTAMIBI scan and weakly positive on FDG-PET scan and a $7 \mathrm{~mm}$ right lobe nodule. Left thoracotomy revealed a pleural based neuroendocrine tumor with lympho-vascular and lung parenchyma invasion, three additional intrapulmonary lesions, and a hilar mass.

All six lesions were surgically resected and stained positive for synaptophysin and negative for chromogranin A and TTF-1, features consistent with neuroendocrine tumor. Intraoperative PTH fell by $\sim 70 \%$ after tumor resection supporting the diagnosis of parathyroid cancer. Though lowered, calcium and PTH levels remained persistently elevated at and after discharge (August 5, 2007) (Fig. 1).

Due to persistent hypercalcemia and lack of other treatment options, the patient was offered and consented to receive anti-hPTH immunotherapy treatment administered in Brazil starting July 2007. Initial investigation was approved by the University Ethics Committee (ETIC 367/ 07). Further approval requirements for the immunization protocol were considered as compassionate care considering that no other clinically proven, effective treatments were available; the protocol was explained in detail to the patient, was carried out free of charge, and the patient gave written informed consent to the protocol as previously published [9].

Anti-hPTH immunogen was administered monthly, for 5 months. Each immunization was distributed over seven sites, targeting the afferent drainage areas of major lymph node groups, as directed. Irritating BCG-like irritating ulcers developed in the skin at injection sites and healed over a period of 8 weeks. Dot-blot assays assessing immune response of anti-hPTH antibody (after the first immunization) showed strong reaction to the N-terminal (1-34) peptide, moderate reaction to the C-terminal (51-84) peptide, and weak reaction to the mid-section (33-52) peptide (data not shown). After five immunizations, calcium and PTH levels were still elevated and rising (Fig. 1) and were attributed to a combination of tumoral cell disruption (calcium rise) and to immunogen cross reactivity (PTH rise). Chest CT revealed multiple novel thoracic lesions not visualized before immunotherapy (Fig. 2). Nine resectable lung lesions were surgically removed, each showing granulomatous inflammation surrounding parathyroid metastatic lesions (Fig. 3).

PTH and calcium levels were lowered to within or below normal limits 16 days after surgery (November 26, 2017) and remained in that range continuously until last follow up (February 2019) (Fig. 1). In September 2019, 12 years after last immunotherapy, she was asymptomatic, on daily replacement with cholecalciferol (400 IU), calcium carbonate $(500 \mathrm{mg}$ calcium), and L-thyroxine $(112 \mu \mathrm{g})$. Her PTH level was $6.1 \mathrm{pg} / \mathrm{mL}$, ionized calcium $1.13 \mathrm{mmol} / \mathrm{L}$, total calcium $2.0 \mathrm{mM}$, anti-TPO $0.6 \mathrm{IU} / \mathrm{mL}(<9.0 \mathrm{IU} / \mathrm{mL}), 25$ Hydroxycholecalciferol $57 \mathrm{ng} / \mathrm{mL}$, and antithyroglobulin $25 \mathrm{IU} / \mathrm{mL}(<27 \mathrm{IU} / \mathrm{mL})$. FDG-PET/CT performed in March 2019 showed no evidence of disseminated parathyroid carcinoma. Her PTHrP levels were measured in September 2019 and was undetectable $(N<1.02 \mathrm{pmol} / \mathrm{L})$. Immunoglobulins were all within normal values. In addition, her medical history was negative for recurring infections, as an indirect measurement of an inadequate immune system.

\section{Discussion}

We report a 12-year remission in disseminated parathyroid carcinoma [13] after combined immunotherapy and surgical treatment. While the case reported herein presents the longest remission to date of metastatic parathyroid cancer in response to combined immunotherapy and surgery, it is hard to assign the relative contribution of each of these two therapeutic modalities to this favorable clinical outcome. To date, complete surgical excision is the only potentially curative therapeutic option but has only been possible for disease confined locally to the neck $[2,3,7,14]$. However, surgery alone has consistently failed to lead to long-term survival in disseminated disease. The mechanisms that may render parathyroid carcinoma responsive to immunotherapy are unclear. Immunity against the entire PTH molecule seems essential. However, this feature alone cannot account for the antitumoral effect with loss of parathyroid cancer tissues. Loss of paracrine growth promoting effect of PTH 
Fig. 1 a Total calcium (reference $8.3-10.6 \mathrm{mg} / \mathrm{dL}$ ) and b total PTH (reference $18.5-88.0 \mathrm{pg} / \mathrm{mL}$ ) values over time. Normal range is shown in gray. The high levels of PTH during and immediately post immunization are attributed to cross reactivity with the immunogen. Dotted lines point to all five courses of immunization (IM), and surgical procedure (S) to remove newly discovered lesions in Brazil (11/ 26/2007) after previous surgery (May 2007) at the NIH
A

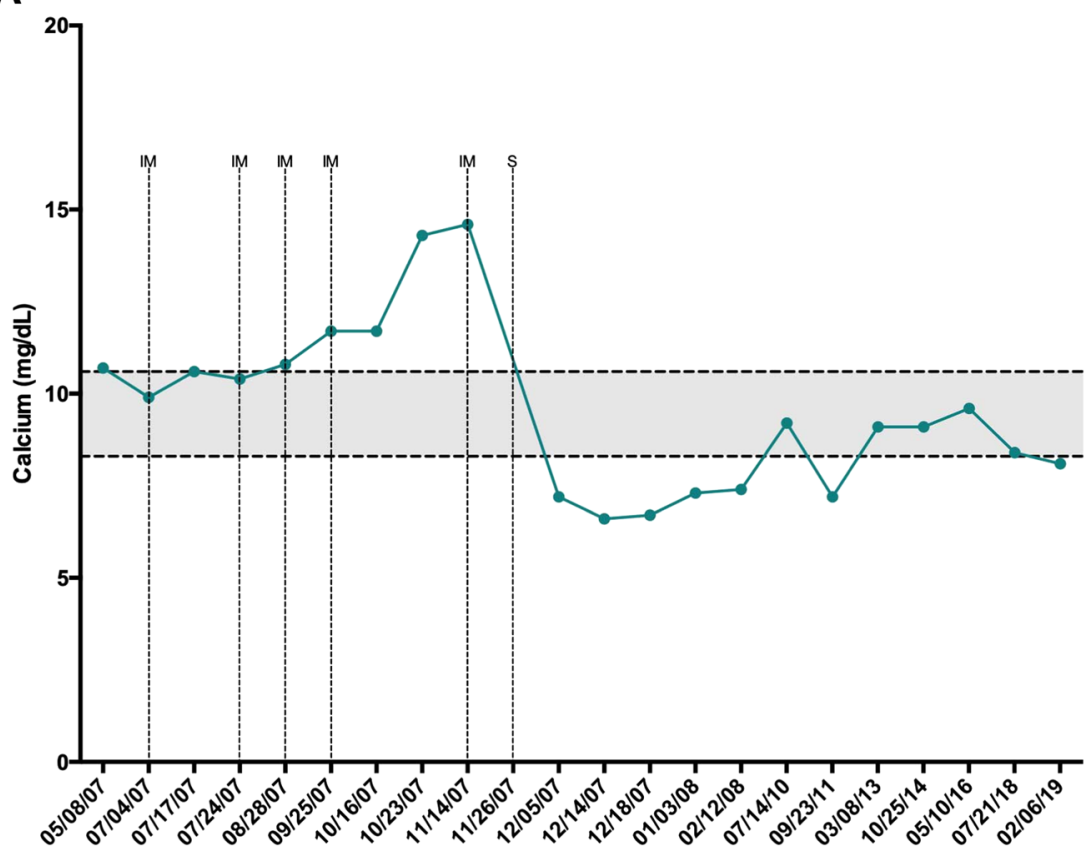

B

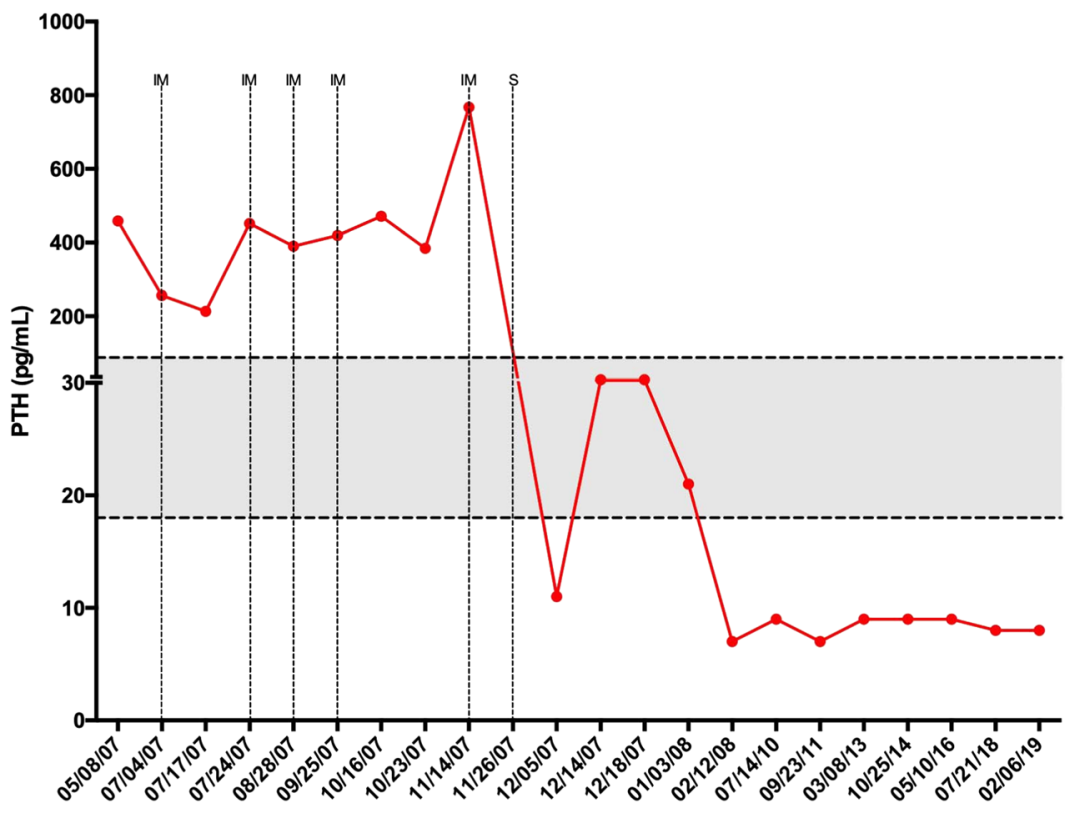

on its own cells could contribute, but there is no evidence for such mechanism in parathyroid tissues. Clarke et al. [15] showed that the genomic landscape of parathyroid carcinoma involves somatic mutations in genes mediating immune response (e.g., PTPRB). This finding may highlight the putative response mechanism of parathyroid carcinoma to immunomodulation. Indeed, Piranavan et al. [16] recently showed that a checkpoint inhibitor could evoke antibodies that inhibit secretion of PTH; perhaps a similar mechanism might evoke cytotoxic antibodies against the parathyroid. Immunotherapy may have caused an inflammatory reaction that rendered parathyroid carcinoma metastases visible on imaging. This effect could be due to the Freund's complete adjuvant, the most potent adjuvant for inducing high titer antibodies (via Th2 responses) and a very effective agent for inducing Th1 and CD8 cytotoxic Tcell responses. This finding facilitated their surgical removal and may have contributed to a lasting remission. However, it seems unlikely that this mechanism could solely account for removal of all metastases, rendering the patient hypoparathyroid. Lastly, we have not excluded the possibility that the immunogenic benefit was attributable to complete 
Fig. 2 High resolution chest CT. a $2.5 \mathrm{~cm}$ noncalcified left apical multilobulated mass (2007). b $7 \mathrm{~mm}$ right lobe nodule, (2007). c Absence of right lobe nodule (2019). d $7.5 \mathrm{~mm}$ postsurgical residual fibrotic striae (2019), which have been stable since 2008
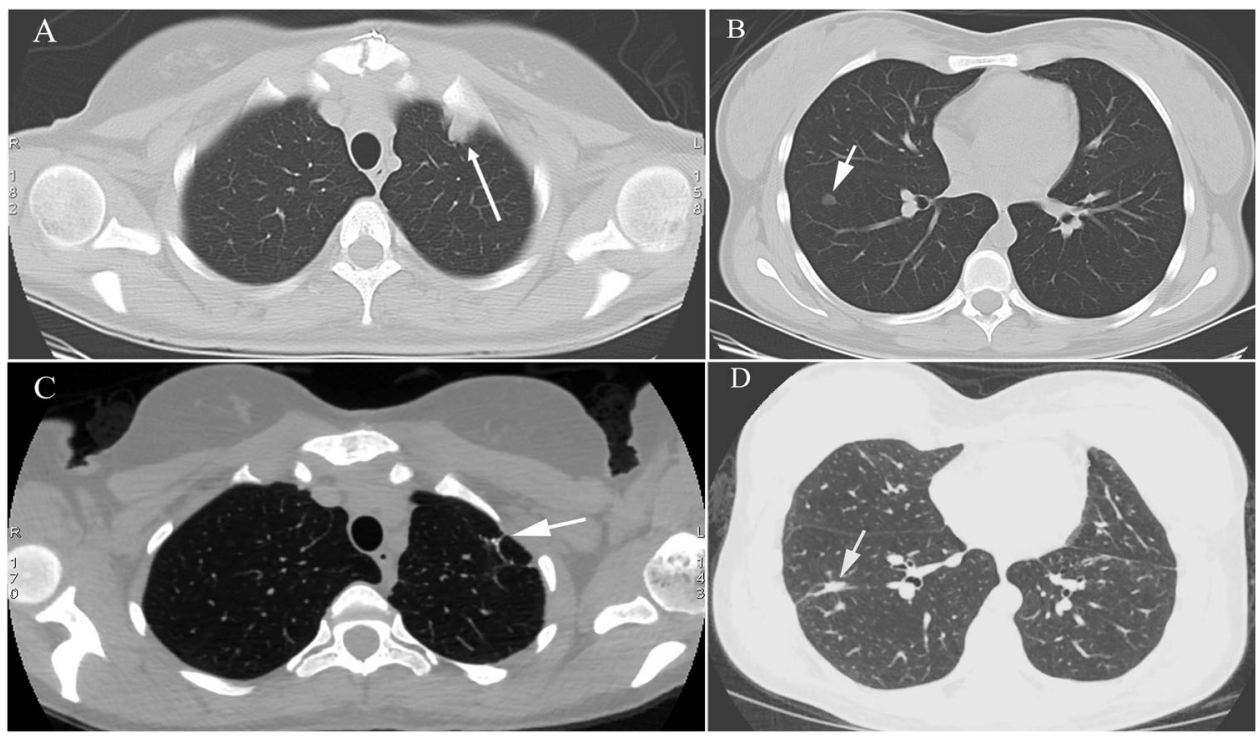

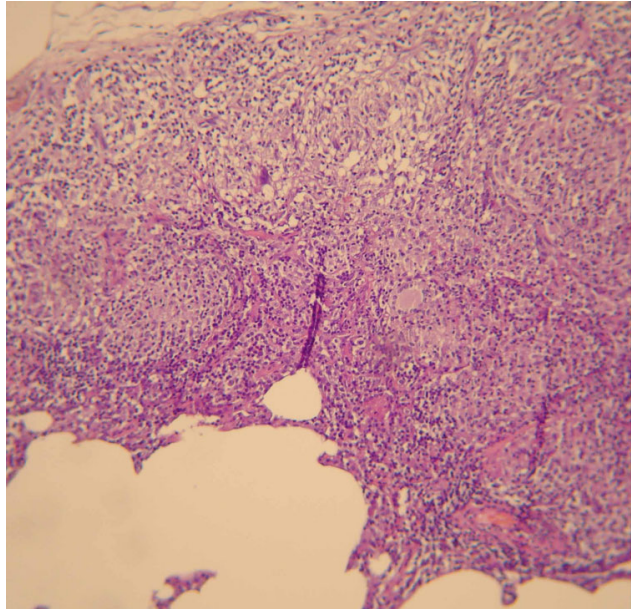

Fig. 3 Fragment of pulmonary tissue, showing chronic granulomatous inflammation with multinucleated giant cells. Parathyroid cancer cells are not shown in this image (image not available)

Freund's adjuvant alone and independent of the PTH component; however, there is no past precedent for such a benefit of complete Freund's in the overall cancer literature.

Bradwell et al. [9] first reported the efficacy of PTH immunization in a 62-year-old woman with parathyroid carcinoma, multiple lung and pleural metastases, severe bone disease, and extreme hypercalcemia. Another 50year-old Belgian patient with metastatic parathyroid carcinoma received eight courses of anti-PTH immunization [10]. After the fourth immunization, both calcium and PTH started decreasing and a $40-70 \%$ decrease in pulmonary metastases size was noted with concomitant improvement of the patient's clinical condition for more than 24 months [10]. A Japanese female with multiple parathyroid carcinoma metastases received anti-PTH monthly immunizations. Despite achieving high antibody titers and lowering of calcium levels, the patient died after the sixth course of immunization due to tumor progression [11].

Our patient had a prolonged interval with consistently low to normal serum calcium and low PTH suggesting a hypoparathyroid state. The origin of the low but detectable PTH is of interest. It is unlikely from residual carcinoma tissue since it remained stable over time without further therapy. It might be from residual normal but insufficient parathyroid tissue. It could also be from cross reactivity with a similar peptide (e.g., PTHrP). However, this is unlikely as measurements of this latter peptide were undetectable. Lastly, it could represent true aparathyroidism plus the noise signal from undetectable PTH.

The case presented herein adds to the accumulating body of evidence that immunotherapy should be explored as a valid therapeutic option in patients with metastatic and particularly disseminated, parathyroid carcinoma.

Acknowledgements We thank the members of the NIH Clinical Center for their involvement in treating this patient and the Medical team at the Centro de Tecnologia e Medicina Molecular, Faculdade de Medicina/UFMG, in Belo Horizonte, Brazil.

Funding This work was partially funded by grant from Conselho Nacional de Desenvolvimento Científico e Tecnológico ( $\mathrm{CNPq} \#$ 405053/2013-4).

\section{Compliance with ethical standards}

Conflict of interest The authors declare that they have no conflict of interest.

Publisher's note Springer Nature remains neutral with regard to jurisdictional claims in published maps and institutional affiliations. 


\section{References}

1. C. Sadler, C. Sadler, K.W. Gow, E.A. Beierle, J.J. Doski, M. Langer, J.G. Nuchtern, S.A. Vasudevan, M. Goldfarb, Parathyroid carcinoma in more than 1,000 patients: a population-level analysis. Surgery 156, 1622-1629 (2014)

2. D. Betea, I. Potorac, A. Beckers, Parathyroid carcinoma: challenges in diagnosis and treatment. Ann. Endocrinol. 76, 169-177 (2015)

3. A.S. Salcuni, F. Cetani, V. Guarnieri, V. Nicastro, E. Romagnoli, D. de Martino, A. Scillitani, D.E.C. Cole, Parathyroid carcinoma. Best Pract. Res. Clin. Endocrinol. Metab. 32, 877-889 (2018)

4. K. Brewer., J. Costa-Guda, A. Arnold, Molecular genetic insights into sporadic primary hyperparathyroidism. Endocr. Relat. Cancer 26, R53-R72 (2019)

5. E. Cavalier, A.F. Daly, D. Betea, P.N. Pruteanu-Apetrii, P. Delanaye, P. Stubbs, A.R. Bradwell, J.P. Chapelle, A. Beckers, The ratio of parathyroid hormone as measured by third- and second-generation assays as a marker of parathyroid carcinoma. J. Clin. Endocrinol. Metab. 95, 3745-3749 (2010)

6. A. Harari, A. Waring, G. Fernandez-Ranvier, J. Hwang, I. Suh, E. Mitmaker, W. Shen, J. Gosnell, Q.Y. Duh, O. Clark, Parathyroid carcinoma: a 43-year outcome and survival analysis. J. Clin. Endocrinol. Metab. 96, 3679-3686 (2011)

7. E.A. Asare, C. Sturgeon, D.J. Winchester, L. Liu, B. Palis, N.D. Perrier, D.B. Evans, D.P. Winchester, T.S. Wang, Parathyroid carcinoma: an update on treatment outcomes and prognostic factors from the National Cancer Data Base (NCDB). Ann. Surg. Oncol. 22, 3990-3995 (2015)

8. Y. Takeuchi, S. Takahashi, D. Miura, M. Katagiri, N. Nakashima, H. Ohishi, R. Shimazaki, Y. Tominaga, Cinacalcet hydrochloride relieves hypercalcemia in Japanese patients with parathyroid cancer and intractable primary hyperparathyroidism. J. Bone Min. Metab. 35, 616-622 (2017)

9. A.R. Bradwell, T.C. Harvey, Control of hypercalcaemia of parathyroid carcinoma by immunisation. Lancet 353, 370-373 (2019)
10. D. Betea, A.R. Bradwell, T.C. Harvey, G.P. Mead, H. SchmidtGayk, B. Ghaye, A.F. Daly, A. Beckers, Hormonal and biochemical normalization and tumor shrinkage induced by antiparathyroid hormone immunotherapy in a patient with metastatic parathyroid carcinoma. J. Clin. Endocrinol. Metab. 89, 3413-3420 (2014)

11. I. Horie, T. Ando, N. Inokuchi, Y. Mihara, S. Miura, M. Imaizumi, T. Usa, N. Kinoshita, I. Sekine, S. Kamihara, K. Eguchi, First Japanese patient treated with parathyroid hormone peptide immunization for refractory hypercalcemia caused by metastatic parathyroid carcinoma. Endocr. J. 57, 287-292 (2010)

12. V.M. Howell, C.J. Haven, K. Kahnoski, S.K. Khoo, D. Petillo, J. Chen, G.J. Fleuren, B.G. Robinson, L.W. Delbridge, J. Philips, A. E. Nelson, U. Krause, K. Hammje, H. Dralle, C. Hoang-Vu, O. Gimm, D.J. Marsh, H. Morreau, B.T. The, HRPT2 mutations are associated with malignancy in sporadic parathyroid tumours. J. Med Genet. 40, 657-663 (2003)

13. M.S. Sarquis, L.G. Silveira, F.J. Pimenta, E.P. Dias, B.T. Teh, E. Friedman, R.S. Gomez, G.C. Tavares, C. Eng, L. De Marco, Familial hyperparathyroidism: surgical outcome after 30 years of follow-up in three families with germline HRPT2 mutations. Surgery 43, 630-640 (2008)

14. L. Rozhinskaya, E. Pigarova, E. Sabanova, E. Mamedova, I. Voronkova, J. Krupinova, L. Dzeranova, A. Tiulpakov, V. Gorbunova, N. Orel, A. Zalian, G. Melnichenko, I. Dedov, Diagnosis and treatment challenges of parathyroid carcinoma in a 27 -yearold woman with multiple lung metastases. Endocrinol. Diabetes Metab. Case Rep. 6, 0113 (2017)

15. C.N. Clarke, P. Katsonis, T.K. Hsu, A.M. Koire, A. Silva-Figueroa, I. Christakis, M.D. Williams, M. Kutahyalioglu, L. Kwatampora, Y. Xi, J.E. Lee, E.S. Koptez, N.L. Busaidy, N.D. Perrier, O. Lichtarge, Comprehensive genomic characterization of parathyroid cancer identifies novel candidate driver mutations and core pathways. J. Endocr. Soc. 3, 544-559 (2018)

16. P. Piranavan, Y. Li, E. Brown, E.H. Kemp, N. Trivedi, Immune checkpoint inhibitor-induced hypoparathyroidism associated with Calcium-Sensing Receptor-Activating autoantibodies. J. Clin. Endocrinol. Metab. 104, 550-556 (2019) 